\title{
Molecular genetics and phenotype of 26 Vietnamese patients with congenital hyperinsulinism
}

\author{
Vu Chi Dung ${ }^{1 *}$, Nguyen Thanh Liem", Bui Phuong Thao ${ }^{1}$, Nguyen Ngoc Khanh' ${ }^{1}$, Can Thi Bich Ngoc', \\ Nguyen Thi Hoan ${ }^{1}$, Khu Thi Khanh Dung ${ }^{1}$, Le To Nhu' ${ }^{1}$, Dang Anh Duong ${ }^{1}$, Nguyen Phu Dat ${ }^{1}$, Sarah Flanagan², \\ Sian Ellard ${ }^{2}$
}

From 7th APPES Biennial Scientific Meeting

Nusa Dua, Bali. 14-17 November 2012

Hyperinsulinemic hypoglycemia $(\mathrm{HH})$ is a consequence of unregulated insulin secretion by pancreatic $\beta$-cells and is a major cause of hypoglycemic brain injury and mental retardation. Congenital HH is caused by mutations in genes involved in regulation of insulin secretion, seven of which have been identified $(A B C C 8, K C N J 11$, GLUD1, CGK, HADH, SLC16A1 and HNF4A). Severe forms of congenital $\mathrm{HH}$ are caused by mutations in $A B C C 8$ and $K C N J 11$, which encode the two components of the pancreatic $\beta$-cell ATP-sensitive potassium channel (sulfonylurea receptor SUR1 and the inwardly rectifying ion channel KIR6.2). Activating mutations in the subunit genes of ATP-sensitive potassium channel can result in monogenic diabetes, whereas inactivating mutations are the most common cause of congenital hyperinsulinism of infancy.

We aim to identify mutations of ABCC8; KCNJ11 and HNF4A in Vietnamese patients with congenital $\mathrm{HH}$, to describe the phenotype, and to evaluate outcome of these patients.

This is a case series study including phenotype, genotype characteristics and outcome. Twenty six Vietnamese probands with congenital $\mathrm{HH}$ were analyzed for alterations in $A B C C 8 ; K C N J 11$ and HNF4A. All exons of KCNJ11; $A B C C 8$ and HNF4A genes were amplified from genomic DNA and directly sequenced. In patients with detected mutations, the parental origin of each mutation was determined. $13 / 26$ cases with no identified mutations of $A B C C 8$; KCNJ11 and HNF4A were stable with medical treatment (diazoxide-responsive congenital $\mathrm{HH}$ ). Eleven probands had mutations in the $A B C C 8$ and no mutation in the KCNJ11 and HNF4A. Six patients were homozygous or compound heterozygous for the mutations, indicating diffuse pancreatic disease. Their blood glucose levels were normal after nearly total pancreatectomy by laparoscopy. In five patients, heterozygous and paternally inherited mutations were found, suggesting focal disease before surgery. Of which one case had diffuse lesions on histopathology examination and normal blood glucose level after pancreatectomy, one case had diabetes after two days of surgery and stop of insulin injection after 6 months of treatment and one case still needs octreotide treatment for normal blood glucose levels. Altogether, 9 different ABCC 8 mutations including three novel alterations (p.F686I, p.I395F and p.G1379S) and six reported mutations (p.F686S, IVS27-1G>A, p.R999X, c.1467+5G>A, p.R934X and p.S1387del) were identified. One case of responsive with diazoxide had partenal inherited mutation in KCNJ11 [c.482C>T (p.A161V)] and one case of responsive with diazoxide had a novel mutation and maternal inheritance in HNF4A [c.659T>C (p.L220P)]. Our results extend the knowledge of the molecular genetics, phenotype and outcome behind congenital HH in Vietnam.

\section{Authors' details \\ 'Vietnam National Hospital of Pediatrics, Hanoi, Vietnam. ${ }^{2}$ Peninsula Medical School, Institute of Biomedical and Clinical Science, Exeter, UK.}

Published: 3 October 2013

\section{doi:10.1186/1687-9856-2013-S1-P179}

Cite this article as: Dung et al:: Molecular genetics and phenotype of 26 Vietnamese patients with congenital hyperinsulinism. International Journal of Pediatric Endocrinology 2013 2013(Suppl 1):P179. 